Abstract

\title{
On Conditional Tsallis Entropy ${ }^{\dagger}$
}

\author{
Andreia Teixeira 1,2,3, André Souto 4,5,6 and Luís Antunes 7,8 \\ 1 Centre for Health Technology and Services Research (CINTESIS), Faculty of Medicine University of Porto, \\ Porto, Portugal \\ 2 Health Information and Decision Sciences Department-MEDCIDS, Faculty of Medicine, University of Porto, \\ Porto, Portugal \\ 3 ARC4DigiT, Polytechnic Institute of Viana do Castelo, Viana do Castelo, Portugal \\ 4 Departamento de Informática Faculdade de Ciências da Universidade de Lisboa, Lisboa, Portugal \\ 5 LASIGE, Universidade de Lisboa, Lisboa, Portugal \\ 6 Instituto de Telecomunicações, Aveiro, Portugal \\ 7 Institute for Systems and Computer Engineering, Technology and Science (INESC-TEC), Porto, Portugal \\ 8 DCC-FCUP, University of Porto, Porto, Portugal \\ + Presented at the Entropy 2021: The Scientific Tool of the 21st Century, 5-7 May 2021; Available online: \\ https://sciforum.net/conference/Entropy2021/.
}

Published: 5 May 2021

Tsallis entropy, a generalisation of Shannon entropy that depends on a parameter alpha, provides an alternative way of dealing with several characteristics of nonextensive physical systems given that the information about the intrinsic fluctuations in the physical system can be characterized by the nonextensivity parameter alpha. It is known that as the parameter alpha approaches 1 , the Tsallis entropy corresponds to the Shannon entropy. Unlike for Shannon entropy, but similarly to Rényi entropy (yet another generalisation of Shannon entropy that also depends on a parameter alpha and converges to Shannon entropy when alpha approaches 1 ), there is no commonly accepted definition for the conditional Tsallis entropy. In this work, we revisit the notion of conditional Tsallis entropy by studying some natural and desirable properties in the existing proposals: when alpha tends to 1, the usual conditional Shannon entropy should be recovered; the conditional Tsallis entropy should not exceed the unconditional Tsallis entropy; and the conditional Tsallis entropy should have values between 0 and the maximum value of the unconditional version. We also introduce a new proposal for conditional Tsallis entropy and compare it with the existing ones.

(C) 2021 by the authors. Licensee MDPI, Basel, Switzerland. This article is an open access article distributed under the terms and conditions of the Creative Commons Attribution (CC BY) license (http://creativecommons.org/licenses/by/4.0/). 